\title{
Application of the High-Gain Substrate-Superstrate Configuration to Dielectric Leaky-Wave Antennas
}

\author{
José Luis Gómez-Tornero, Fernando Quesada-Pereira, and Alejandro Álvarez-Melcón.
}

\begin{abstract}
The high-gain substrate-superstrate configuration, which was proposed to increase the gain in printed circuit antennas, is applied to dielectric leaky-wave antennas (LWAs) to improve its frequency response. Analysis of a slitted suspended dielectric rectangular waveguide is carried out using a full-wave method. It is proved that the minimum values of the leakage constant of the leaky-wave mode for the suspended configuration are related to the high-gain resonance conditions. Moreover, it is found that the suspended LWA exhibits very small beamwidth variations in a large frequency bandwidth. It is well-known that inhomogeneous filled LWAs suffer from variation of beamwidth as the angle of maximum radiation is scanned with frequency. The proposed topology can be adjusted so that a flat response of the beamwidth can be obtained in a large frequency band, while maintaining the frequency-scanning behavior of the LWA.
\end{abstract}

Index Terms-Leaky-wave antennas (LWAs), millimeter-wave antennas.

\section{INTRODUCTION}

$\mathbf{L}$ EAKY-WAVE antennas (LWA) are a type of travelingwave antennas based on the excitation of a leaky-wave mode in an open waveguide. Many interesting features make this type of antenna useful in the millimeter waveband range, as the introduction of low-losses, very large bandwidths, simple fabrication and easy interconnection with standard waveguide and printed technologies [1]. Besides, the radiated beamwidth can be made very small, obtaining high gains while the beam direction can be scanned with the frequency from near broadside to endfire. LWA can be completely hollow or contain a dielectric guide. Air-filled LWA present lower losses than dielectric-filled LWA, but the scanning capability is reduced, not being able to reach the endfire direction. On the contrary, dielectric-filled LWA can scan up to endfire, but have an important disadvantage when compared to air-filled LWA: the beamwidth varies as the beam is scanned with the frequency [1]. This phenomenon can be a disadvantage for many applications, where the same beamwidth is required for different pointing directions.

This letter presents a novel and simple suspended topology, which is applied to a slitted dielectric rectangular waveguide

Manuscript received July 6, 2004; revised October 7, 2004. This work was supported by Spanish National Projects ESP2001-4546-PE and TIC2000-0591C03-03, by Regional Seneca Projects 2002 PB/4/FS/02 and PMPDI-UPCT2004, and by the EPSON-Ibérica Foundation. The review of this letter was arranged by Associate Editor M. Mrozowski.

The authors are with Departamento de Tecnologías de la Información y las Comunicaciones, Technical University of Cartagena, Cartagena 30202, Spain (e-mail: josel.gomez@upct.es).

Digital Object Identifier 10.1109/LMWC.2005.845731

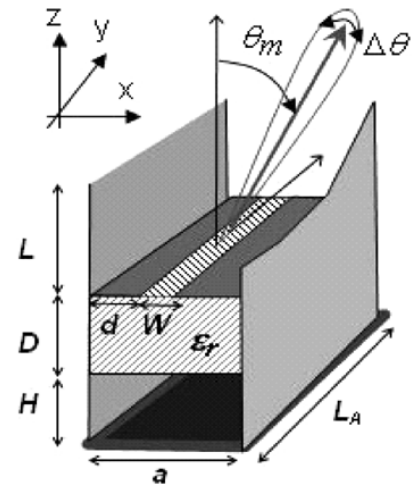

\begin{tabular}{|l|}
\hline$a=2.25 \mathrm{~mm}$ \\
$D=1.59 \mathrm{~mm}$ \\
$L=1 \mathrm{~mm}$ \\
$\varepsilon r=2.56$ \\
$W=0.6 \mathrm{~mm}$ \\
$d=0.2 \mathrm{~mm}$ \\
$f=50 G \mathrm{~Hz}$ \\
\hline
\end{tabular}

Fig. 1. Suspended dielectric LWA.

(Fig. 1), previously studied in [2], to improve the frequency response of the leaky-wave mode. It is shown that the new structure synthesizes a flat response for the beamwidth in a large frequency band. This phenomenon can be related to the high-gain substrate-superstrate configuration presented in [3], devoted to improve the gain in printed circuit antennas [3]-[5]. LWA do not suffer from low gains, but this resonant high-gain configuration allows minimizing the variation of the beamwidth for inhomogeneous filled LWA, as it will be demonstrated.

\section{THEORY AND RESULTS}

A full-wave, accurate method of moment (MoM) technique [6], [7] is used to analyze the effect of the suspended configuration in the slitted dielectric rectangular guide LWA shown in Fig. 1. In the standard configuration [2], the rectangular dielectric guide, of height $D$ and width $a$, is not suspended from the bottom plane $(H=0)$. The rectangular dielectric guide dimensions $(a, D)$, determine the propagation characteristics of the leaky-wave mode which must be responsible for the radiation of this antenna. This leaky-wave mode is basically the $\mathrm{TE}_{10}$ mode of the dielectric rectangular waveguide (the index " 1 " corresponds to dimension " $a$ "), but perturbed by the slot located at the top wall in order to make it radiate, and also to control the radiated beamwidth. The leaky-wave mode has a complex propagation constant in the longitudinal direction of the open waveguide

$$
k_{y}=\beta-j \alpha .
$$

Fig. 2 shows the variation with frequency of the phase $(\beta)$ and attenuation $(\alpha)$ constants for the leaky-wave mode of the nonsuspended LWA $(H=0 \mathrm{~mm})$. From the plot of $\beta$, it can be seen that the leaky-wave mode is a perturbation of the $\mathrm{TE}_{10}$ mode of 

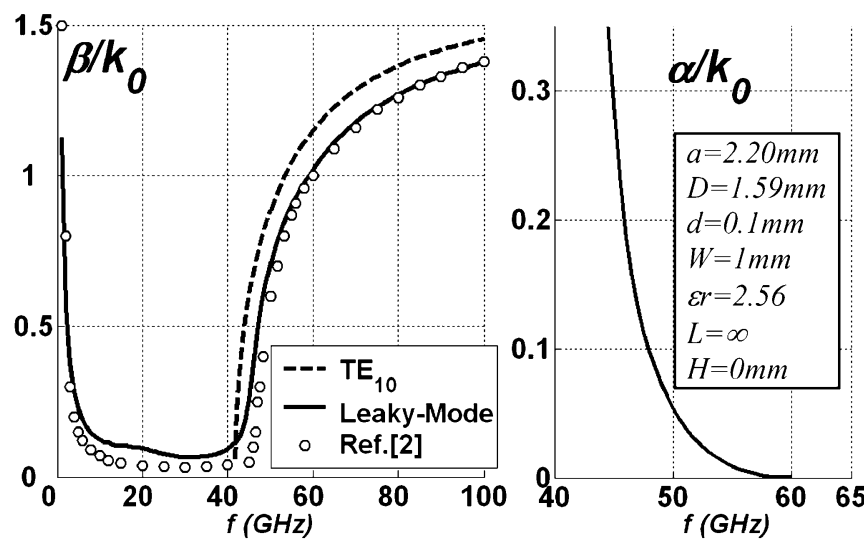

Fig. 2. Phase and attenuation constant for the nonsuspended LWA

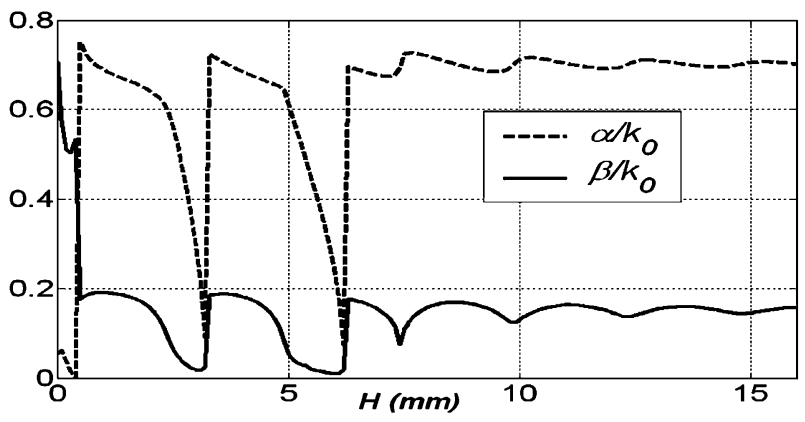

Fig. 3. Modification of $\beta$ and $\alpha$ with the suspended configuration.

the dielectric guide; results from [2] are also plotted for comparison purposes (note that in [2] $L$ is assumed to be infinite). The attenuation constant $\alpha$ decreases as the frequency increased, from large values of $\alpha$ below the cut-off region $(45 \mathrm{GHz})$ to zero for the surface-wave regimen (above $60 \mathrm{GHz}$ ).

From $\beta$ and $\alpha$, the beam direction $\left(\theta_{m}\right)$ and the $3-\mathrm{dB}$ beamwidth $(\Delta \theta)$ of the antenna can be approximately computed with the next known formulas [1] (where the antenna length is assumed so that $90 \%$ of the injected power is radiated)

$$
\begin{aligned}
\sin \theta_{m} & \cong \frac{\beta}{k_{0}} \\
\triangle \theta & \cong \frac{\frac{\alpha}{k_{0}}}{0.189 \cdot \cos \theta_{m}} .
\end{aligned}
$$

When the suspended configuration is applied $(H \neq 0 \mathrm{~mm})$, the leaky-wave mode is perturbed, showing the behavior plotted in Fig. 3. It can be seen some minimums of $\alpha$ at some given values of $H$.

This behavior is due to the high-gain resonance phenomenon, described in [3], and more recently related in [8] to the excitation of weakly attenuated leaky-waves. It was demonstrated in [3] that a narrow beam can be obtained at any desired angle $\theta_{m}$ using a two layered topology, if the next resonance conditions are satisfied

$$
\begin{aligned}
& \sqrt{\varepsilon_{r 1}} \frac{H}{\lambda_{0}} \sqrt{\frac{1-\sin ^{2} \theta_{m}}{\varepsilon_{r 1}}}=\frac{m}{2} \\
& \sqrt{\varepsilon_{r 2}} \frac{D}{\lambda_{0}} \sqrt{\frac{1-\sin ^{2} \theta_{m}}{\varepsilon_{r 2}}}=\frac{2 p-1}{2}
\end{aligned}
$$

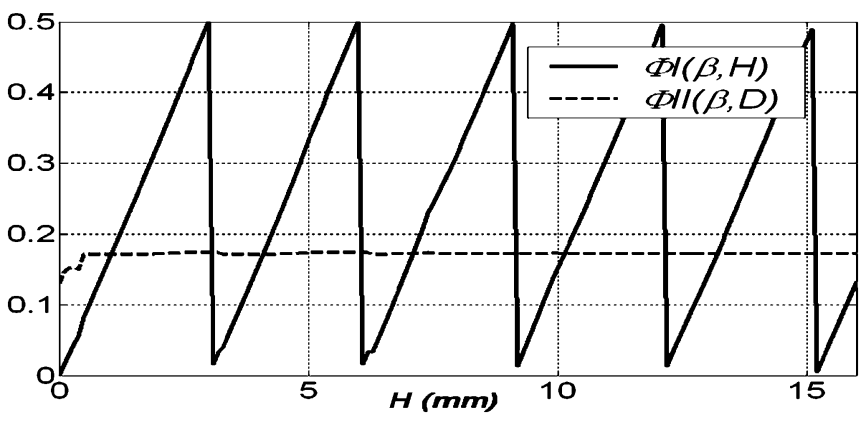

Fig. 4. Resonance Conditions for the suspended LWA.

being $m$ and $p$ positive integers, and obtaining a narrower beam (higher gains) at $\theta_{m}$ as $\epsilon_{r 2} \gg 1$. In our case, the proposed suspended configuration uses $\epsilon_{r 1}=1$ and $\epsilon_{r 2}=\epsilon_{r}=2.56$ (see Fig. 1). In [8], a leaky-wave analysis was used to explain this narrow-beam phenomenon. There, it was demonstrated that the resonance gain was attributable to the excitation of weakly attenuated leaky waves on the structure, which were responsible for the radiation, therefore leading to a narrow radiated beam. To check that the minimums of $\alpha$ shown in Fig. 3 are related to the resonance conditions, (4) and (5) are rewritten using (2)

$$
\begin{aligned}
F I(\beta, H) & =\frac{H}{\lambda_{0}} \sqrt{1-\left(\frac{\beta}{k_{0}}\right)^{2}}=\frac{m}{2} \\
F I I(\beta, D) & =\sqrt{\varepsilon_{r}} \frac{D}{\lambda_{0}} \sqrt{1-\left(\frac{\beta}{k_{0}} \frac{1}{\sqrt{\varepsilon_{r}}}\right)^{2}}=\frac{(2 p-1)}{2} .
\end{aligned}
$$

In order to plot the values of $H$ for which these resonance equations are satisfied, it is easier to use the next functions, which are equal to zero at the resonance conditions

$$
\begin{aligned}
\Phi I(\beta, H) & =\bmod (F I, 0.5)=0 \\
\Phi I I(\beta, D) & =\bmod (F I I+0.25,0.5)=0 .
\end{aligned}
$$

Using the variation of $\beta$ and $H$ shown in Fig. 3, (8) and (9) can be computed and plotted, obtaining the results shown in Fig. 4.

It can be seen that the minimums of $\alpha$ in Fig. 3 correspond to values of $H$ at which the resonance condition $\Phi I$ is fulfilled, which is the most important condition for the high-gain effect [3]. Therefore, it is verified that the simple suspended configuration allows to obtain the high-gain resonance phenomenon. As commented, there are other ways to obtain high gains in LWA (by weakly attenuating the leaky-wave mode), which are simpler than the proposed suspended configuration [1]. However, the suspended configuration in the high-gain condition can be used to achieve a completely novel and very interesting feature.

Fig. 5 shows the frequency response of $\theta_{m}$ and $\Delta \theta$ for the leaky-wave mode for three different values of $H$ and $D$. The original, nonsuspended configuration $(H=0 \mathrm{~mm}, D=$ $1.59 \mathrm{~mm}$, solid line) is compared with the results obtained for the suspended LWA with a value of $H=3 \mathrm{~mm}$ corresponding to the first resonance condition shown in Figs. 3 and 4 ( $H=$ $3 \mathrm{~mm}, D=1.59 \mathrm{~mm}$, dotted line). It can be seen from the plot of $\theta_{m}$, that the response of the antenna has shifted to higher frequencies. The dielectric guide thickness can be readjusted to 

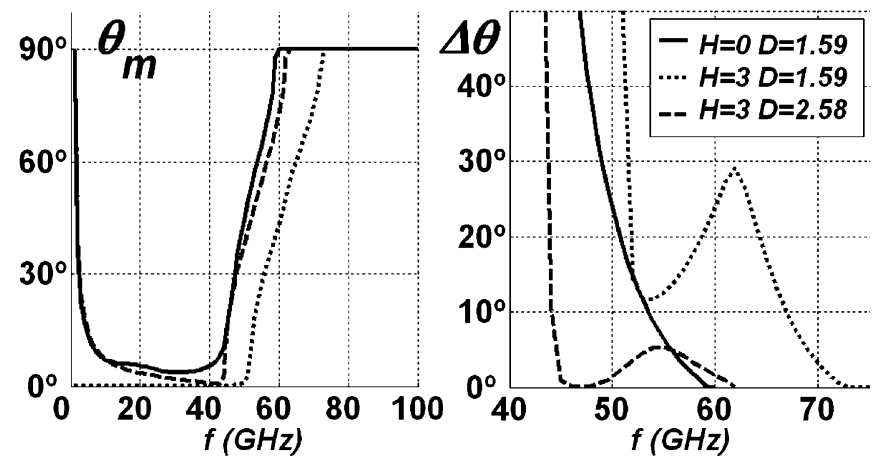

Fig. 5. Frequency response of the LWA for different values of $H$ and $D$ (mm).

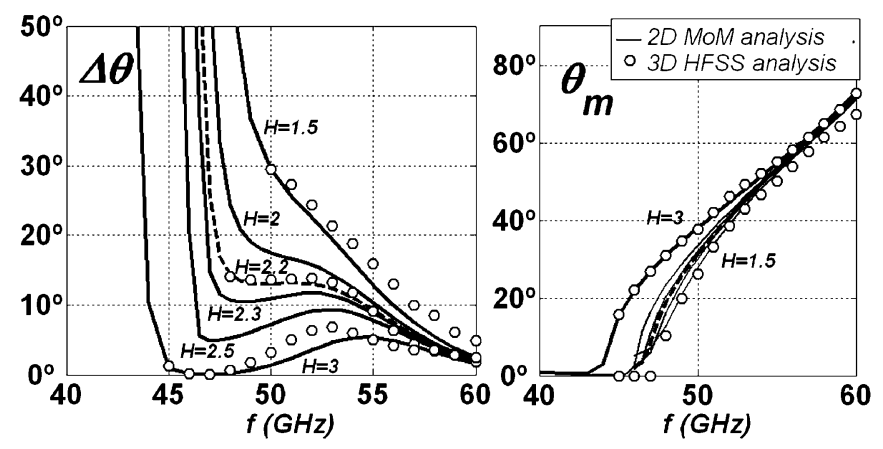

Fig. 6. Optimization of the beamwidth response by varying $H(D=$ $2.58 \mathrm{~mm})$.

center the leaky-wave mode response at $50 \mathrm{GHz}(H=3 \mathrm{~mm}$, $D=2.58 \mathrm{~mm}$, dashed line).

Of much interest is the frequency behavior of the beamwidth $\Delta \theta$, seen in Fig. 5. It can be seen that the standard frequency decreasing curve of $\Delta \theta$ for the nonsuspended LWA changes to a quasioscillatory shape for the high-gain configuration. This is due to the resonant nature of this arrangement, showing a valley at a given frequency, which is followed by a peak. For lower frequencies $(f<45 \mathrm{GHz}), \alpha$ suddenly increases due to the cut-off typical response, leading to high values for $\Delta \theta$, which have no physical meaning since the mode can neither propagate nor radiate. Also, in the surface-wave region $\Delta \theta$ becomes zero since there is no radiation. The radiation region of the LWA is located between these two limits (from $45 \mathrm{GHz}$ to $60 \mathrm{GHz}$ ).

The idea is to use the resonant behavior of the high-gain configuration to obtain a flat response of $\Delta \theta$ in the radiation region of the LWA. Fig. 6 shows that this can be done by adjusting the value of $H$ to an optimum suspension height, which in our case is $H=2.2 \mathrm{~mm}$. Validation results from HFSS 3D simulations are also plotted with circles in Fig. 6, showing good agreement with the results obtained with the two-dimensional (2-D) MoM technique used in this letter. A flat beamwidth response is obtained from $48 \mathrm{GHz}$ to $53 \mathrm{GHz}$, with a nearly constant value of $\Delta \theta \approx 14^{\circ}$, while maintaining unchanged the original LWA scan angle ability for different values of $H\left(\theta_{m}\right.$ varies from $10^{\circ}$ at $48 \mathrm{GHz}$ to $43^{\circ}$ at $53 \mathrm{GHz}$ ). Below and above the optimum value of $H$, the response of $\Delta \theta$ is either too oscillatory or it decreases linearly with a too step slope, respectively. It can be seen that a second-order effect occurs when varying $H$; this is that the cut-off frequency of the leaky-wave mode is lowered as $H$ is increased. This can be corrected by modifying $D$ to center the response back to the desired frequency band, as previously done.

\section{CONCLUSION}

In this letter, it has been shown that the beamwidth-frequency response of a dielectric LWA can be strongly modified by using a simple suspended configuration. This arrangement can make the leaky-wave mode work in a high-gain resonance condition, obtaining an oscillatory response for its beamwidth as the frequency is varied. By adjusting the thickness of the dielectric guide and the height of the suspension region, the beamwidth variation can be minimized in a certain frequency band, obtaining a constant beamwidth for a wide range of scanning angles. This is an important new feature for dielectric-filled LWAs, since it is desirable in many applications to maintain the same beamwidth for different beam scan-angles.

\section{REFERENCES}

[1] A. A. Oliner, "Leaky-wave antennas," in Antenna Engineering Handbook, 3rd ed, R. C. Johnson, Ed. New York: McGraw-Hill, 1993, ch. 10.

[2] P. Lampariello, F. Frezza, and A. A. Oliner, "The transition region between bound-wave and leaky-wave ranges for a partially dielectric-loaded open guiding structure," IEEE Trans. Microw. Theory Tech., vol. 38, no. 12, pp. 1831-1836, Dec. 1990.

[3] D. R. Jackson and N. G. Alexopoulos, "Gain enhancement methods for printed circuit antennas," IEEE Trans. Antennas Propag., vol. 33, no. 9, pp. 976-987, Sep. 1985.

[4] C. S. Lin, S. S. Zhong, J. H. Shi, and Y. Wang, "Gain enhancement technique for microstrip antennas," in Proc. IEEE Antennas Propag., vol. 36, Jul. 1988, pp. 905-910.

[5] X.-H. Shen, G. A. E. Vandenbosch, and A. Van de Capelle, "Study of gain enhancement method for microstrip antennas using moment method," IEEE Trans. Antennas Propag., vol. 43, no. 3, pp. 227-231, Mar. 1995.

[6] J. L. Gómez and A. A. Melcón, "Non-Orthogonality relations between complex-hybrid-modes: An application for the leaky-wave analysis of laterally-shielded top-open planar transmission lines," IEEE Trans. Microw. Theory Tech., vol. 52, no. 3, pp. 760-767, Mar. 2004.

[7] — , "Radiation analysis in the space domain of laterally-shielded planar transmission lines. Part I: Theory," Radio Sci., vol. 39, no. 3, pp. 1-10, Jun. 2004.

[8] D. R. Jackson and A. A. Oliner, "A leaky-wave analysis of the high-gain printed antenna configuration," IEEE Trans. Antennas Propag., vol. 36, no. 7, pp. 905-910, Jul. 1988. 